\title{
Association between complementary and alternative medicine use and prolonged time to conventional treatment among Thai cancer patients in a tertiary-care hospital
}

\author{
Adit Chotipanich $^{\text {Corresp., }}{ }^{\text {, Chulaporn Sooksrisawat }}{ }^{2}$, Benjamabhon Jittiworapan ${ }^{2}$ \\ ${ }^{1}$ The Department of Otolaryngology, Chonburi Cancer Hospital, Department of Medical Services, Ministry of Public Health, Chonburi, Thailand \\ 2 Nursing Unit, Chonburi Cancer Hospital, Department of Medical Services, Ministry of Public Health, Chonburi, Thailand \\ Corresponding Author: Adit Chotipanich \\ Email address: adit_c@ccc.in.th
}

Objectives. The purpose of this study is to investigate the patterns of complementary and alternative medicine use and its association with time to conventional treatment.

Design. A cross-sectional study design was designed.

Setting and participants. The study was performed at the Chonburi Cancer Hospital, with chart reviews and interviews performed for 426 patients with various cancers between May and December 2018

Results. The results indicated that 192 of the 426 patients (45.1\%) reported using complementary and alternative medicines, with herbal products being the most common type. Approximately $34.3 \%$ of these medicines involved unlabeled herbal products with unidentifiable components. The rates of complementary and alternative medicine use were significantly elevated for men and patients with stage IV cancer. The multivariable linear regression analysis of the relationship between factors and the time until conventional treatment revealed that the regression coefficient of the use of complementary and alternative medicine was 56.3 (95\% confidence interval: 27.9-84.6). This coefficient reflected an additional 56.3 days of time until conventional treatment, relative to patients who did not use complementary and alternative medicine.

Conclusions. The present study revealed that complementary and alternative medicine use was fairly common among Thai patients with cancer, and was associated with a prolonged time to receiving conventional treatment. 
1 Association between complementary and alternative

2 medicine use and prolonged time to conventional treatment

3 among Thai cancer patients in a tertiary-care hospital

4

5 Adit Chotipanich ${ }^{1^{*}}$, Chulaporn Sooksrisawat ${ }^{2}$, and Benjamabhon Jittiworapan ${ }^{2}$

6

71 The Department of Otolaryngology, Chonburi Cancer Hospital, Department of Medical

8 Services, Ministry of Public Health, Thailand

92 Nursing Unit, Chonburi Cancer Hospital, Department of Medical Services, Ministry of

10 Public Health, Thailand

11

12 *Corresponding author:

13 Adit Chotipanich

14 The Department of Otolaryngology, Chonburi Cancer Hospital

15 Tel: +66-8-6389-2342 Fax: +66-3878-3995

16 Email: adit_c@ccc.in.th 
19

\section{Abstract}

Objectives. The purpose of this study is to investigate the patterns of complementary and alternative medicine use and its association with time to conventional treatment.

Design. A cross-sectional study design was designed.

Setting and participants. The study was performed at the Chonburi Cancer Hospital, with chart reviews and interviews performed for 426 patients with various cancers between May and December 2018.

Results. The results indicated that 192 of the 426 patients (45.1\%) reported using complementary and alternative medicines, with herbal products being the most common type. Approximately $34.3 \%$ of these medicines involved unlabeled herbal products with unidentifiable components. The rates of complementary and alternative medicine use were significantly elevated for men and patients with stage IV cancer. The multivariable linear regression analysis of the relationship between factors and the time until conventional treatment revealed that the regression coefficient of the use of complementary and alternative medicine was 56.3 (95\% confidence interval: 27.9 84.6). This coefficient reflected an additional 56.3 days of time until conventional treatment, relative to patients who did not use complementary and alternative medicine.

Conclusions. The present study revealed that complementary and alternative medicine use was fairly common among Thai patients with cancer, and was associated with a prolonged time to receiving conventional treatment. 
41

42

43

44

\section{Introduction}

Cancer is the leading cause of death in Thailand, which is also responsible for a majority of the economic burden of disease,${ }^{1}$ with the cancer survival rate in Thailand being lower than in other developed countries. ${ }^{2}$ One factor that contributes to the relatively lower survival rate is a prolonged time until the patient undergoes cancer treatment, ${ }^{3}$ and reducing this time has become a focus of health policy-making in Thailand. ${ }^{4}$ In 2002, Thailand implemented a universal insurance coverage scheme, which has helped reduce the financial barrier to accessing treatment. However, insufficient financing remains a potential threat to healthcare service quality and sufficiency. ${ }^{5}$ Inadequate availability of cancer treatment services is a major, but not exclusive, cause of prolonged wait times for cancer treatment, and many patient-related factors can also delay their accessing healthcare services. For example, impoverishment, distance to the treating center, lack of cancer awareness, and patient preference for complementary and alternative medicine (CAM) may preclude or delay timely access to conventional cancer treatments. ${ }^{6}$

Although CAM is difficult to define, the World Health Organization has loosely defined CAM as "a broad set of health care practices that are not part of that country's own tradition or conventional medicine and are not fully integrated into the dominant health-care system". ${ }^{7}$ The present study focused on CAM treatments that were provided by non-medical personnel and not generally provided at Thai public hospitals. sex, education level, income level, ethnicity, chronic diseases, and cancer. ${ }^{8}$ Moreover, patterns of CAM use can vary between countries, based on geographic factors, religion, 
64 government policy and regulation, and barriers to conventional treatments. ${ }^{9-11}$ Previous

65 studies have revealed that many kinds of CAM can benefit select patients. ${ }^{12,13}$ For example, many herbal medicines can be used in combination with chemotherapy to

67 prevent or ameliorate chemotherapy-related side effects, such as nausea and vomiting ${ }^{14}$ However, improper use of CAM can also cause unexpected interactions with many prescription drugs. ${ }^{15}$ Misleading claims and false advertising for CAM can also lead patients to select CAM instead of conventional therapy, ${ }^{16}$ which can ultimately delay access to conventional therapy.

The use of CAM among patients with cancer has been studied by many researchers around the world. ${ }^{17}$ However, little information is available regarding the relationship between CAM use and accessing conventional therapy among Thai patients with cancer. Thus, the present study aimed to investigate the patterns of CAM use and its association with the time until conventional cancer treatment in Thailand. 
78

79

80

81

82

83

84

85

86

87

\section{Materials and methods}

Study design

This cross-sectional hospital-based study was performed at the Chonburi Cancer Hospital, which is a referral center that treats cancer patients from 7 provinces in eastern Thailand. Patients were non-randomly enrolled using convenience sampling during or after conventional treatment for cancer, with data obtained using face-to-face interviews and medical record reviews between May and December 2018. The inclusion criteria were: any diagnosis of cancer (all types), received conventional cancer treatment within the last year, Thai nationality, age of $\geq 18$ years, and sufficient cognitive function to complete the interview. The exclusion criteria were patients who received treatment for oncologic emergencies and patients who were unable to recall past therapies or related events. The study's protocol was approved by Chonburi Cancer Hospital ethics committee (IRB no. 5/2018), and all participants provided written informed consent prior to their enrollment.

\section{$\underline{\text { Interview and data collection }}$}

The interviews were conducted by five interviewers using a semi-structured interview protocol (Article S1). The interview protocol was reviewed by hospital specialists, and all interviewers successfully completed practice interviews with the principle researchers before interviewing the patients. The interview was described to the patients as "a survey that was designed to assess the use of alternative therapy". Patients were assured that their responses would be kept confidential and would not influence their current treatment. Patients were asked if they had ever used common CAM treatments (traditional medicine, herbal medicine, diet supplements and vitamins, 
101 acupuncture, massage, and meditation), as well as whether they had used "any other

102 therapy not generally provided by clinics and hospitals". Patients who responded that

103 they had used CAM treatments were also asked whether they had used any of these

104 therapies to heal their cancer or alleviate cancer-related symptoms. Patients were

105 categorized in the group that used CAM if the therapy was used with the intent to treat

106 the cancer or was started after the patient became aware of the cancer diagnosis.

107 However, herbal medicines that were approved by the Thai Food and Drug

108 Administration and were used with a doctor's prescription were not considered CAM 109 treatments.

Cancer stage, time until conventional treatment, and conventional treatment

111 details were collected via the medical record reviews. The time until conventional

112 treatment was defined as the interval between the date of diagnosis and the first date of

113 conventional cancer treatment at the Chonburi Cancer Hospital. The date of diagnosis

114 was defined as the day on which the diagnostic biopsy was performed. In cases with

115 treatments performed at both the referring hospital and the Chonburi Cancer Hospital,

116 the time until conventional treatment was calculated starting from the last day of the

117 initial treatment at the referring hospital. Conventional treatments were categorized as

118 surgery, chemotherapy, and radiotherapy, with concurrent chemoradiotherapy

119 categorized as radiotherapy.

120 Classification of the herbal medicines and diet supplements was based on the 121 presence or absence of registration with the Thai Food and Drug Administration. Fresh

122 herbs, decoctions, infusions, and unauthorized commercial products were categorized 123 as "homemade products". 
124 Statistical analysis

125 Data were analyzed using SPSS software (version 17.0; SPSS Inc., Chicago, IL,

126 USA). Pearson's chi-square test was used to compare categorical variables between

127 the groups that did and did not use CAM. Differences were considered statistically

128 significant at $p$-values of $<0.05$. Associations and the influences of various factors on

129 time to conventional treatment were analyzed using univariable and multivariable linear

130 regression analyses. Regression coefficients reflected the additional days of time until

131 conventional treatment, relative to the reference category for each variable. 
133

134

135

136

137

138

139

140

141

142

143

144

145

146

147

148

149

150

151

152

153

154 155

\section{Results}

Between May and December 2018, 426 patients were enrolled, including 192 patients (45.1\%) who reported using CAM. Among the 192 patients, 53 patients (27.6\%) reported using CAM concurrent with or after conventional treatment. Approximately $33.6 \%$ of patients reported that they were satisfied with the CAM therapies, $61.1 \%$ reported no noticeable effect, and $5.4 \%$ reported that they were disappointed with the CAM therapies. Significant differences according to the use or non-use of CAM were observed in sex, cancer stage, and cancer type (Table 1).

Table 2 shows the details regarding the 26 kinds of CAM that were identified. Approximately $34.3 \%$ of these therapies involved unlabeled herbal products with unidentifiable components, and most products were produced as decoctions and infusions. The second most common therapy involved traditional Thai medicine ("Ya Mor Sang" or "Dr. Sang's medicine"), which is provided for free by a registered traditional Thai medicine practitioner. The provider claims that this product is composed of rice hulls and other herbal plants. Lingzhi mushroom was the third most frequently used therapy. Traditional Chinese medicine accounted for $23.1 \%$ of the CAM treatments. None of the patients used meditation or acupuncture.

The patients had a median overall time to conventional treatment of 50 days (interquartile range [IQR]: 34.0-89.0 days). Patients who received radiation therapy had a noticeably longer time to conventional treatment (median: 78 days, IQR: $49.5-112.5$ days) than patients who underwent surgery (median: 41 days, IQR: 29.0-62.0 days) or patients who received chemotherapy (median: 39 days, IQR: 28.0-64.0 days). Among patients who used CAM before conventional treatment, 20 patients $(14.4 \%)$ deliberately 
156 chose to delay conventional treatment and instead selected CAM. These 20 patients

157 were temporarily lost to follow-up, and had a median delay time of 382 days (IQR:

$158321.0-640.0$ days), with a longest wait time of 2,067 days for a patient who had been

159 selected for chemotherapy.

Table 3 shows the median times to conventional treatment and their associations

161 with various factors. The multivariable analyses revealed that a prolonged time to

162 conventional treatment was significantly associated with receiving radiation treatment,

163 stage IV cancer, any use of CAM, and use of CAM before starting conventional

164 treatment (Data S1).

165 
166

167

168

169

170

171

172

173

174

175

176

177

178

179

180

181

182

\section{Discussion}

Thailand is one of a handful of middle income countries that freely provides all conventional cancer treatments to all citizens as part of a universal health coverage scheme. ${ }^{18}$ In this context, delays in receiving appropriate cancer treatments, regardless of their reason, can lead to poorer health outcomes and a higher economic burden. ${ }^{19}$ While some CAM professions and products are regulated by governing bodies in Thailand, most CAM treatments remain informal or unregulated. ${ }^{20}$ Thus, given the availability of many unregulated CAM treatments, it is possible that CAM use might prolong the time to conventional treatment among Thai cancer patients. Very few studies have addressed this issue, ${ }^{21-24}$ and the present study aimed to explore the relationship between CAM use and the time to conventional cancer treatment.

Previous studies have revealed varying prevalence of CAM use among patients with cancer, which ranged from $24 \%$ to $84 \%,{ }^{25-29}$ although this variation may be related to the target population and the definition of CAM that the researchers used. The present study revealed that $45.1 \%$ of the patients reported using CAM, which is moderate relative to the rates from previous studies. However, CAM use was significantly more common among men and patients with stage IV cancer.

Herbal products were the most frequently used CAM, and previous studies have indicated that the classification of herbal medicines and diet supplements should be based on intended use, labeling, preparation, and dosage ${ }^{30}$ However, it would be difficult to distinguish between herbal medicines and diet supplements in the present study, as approximately two-thirds of the treatments involved homemade products without proper labeling or appropriate quality control. Furthermore, many treatments 
189 had been registered as diet supplements, but were used with the intent to treat the

190

191

192

193

194

195

196

197

198

199

200

201

202

203

204

205

206

207

208

209

210

211

patient's cancer. Although many patients reported that use of these treatments helped

alleviate their symptoms, the use of treatments with low quality or at inappropriate dosages can cause unpredictable adverse effects and interactions with conventional treatments. ${ }^{31,32}$ Thus, there is a need to encourage the safe use of homemade herbal medicines among cancer patients.

The time to receiving radiotherapy was longer than the times to receiving surgery and chemotherapy. This is because our hospital is the only facility in the eastern Thailand that provides radiotherapy services, with only 3 teletherapy machines available to serve a population of $5,000,000$ residents. Therefore, access to radiotherapy appears to remain a crucial problem in eastern Thailand.

Using CAM before conventional treatments was also significantly associated with the time to conventional treatment. Among patients who used CAM treatments, 20 patients $(14.4 \%)$ had received appointments for conventional treatments but were temporarily lost to follow-up and instead chose to receive CAM treatments. The delay time in that group was remarkably longer than the times to conventional treatment in the other groups, which significantly affected the average time to conventional treatment in the present study. However, other factors may also explain why patients use CAM before conventional treatment, as many patients used CAM as an interim treatment before they could receive conventional treatment. In addition, a prolonged wait for conventional treatment could increase the likelihood that a patient would select CAM. These factors may help explain the association between CAM use and the prolonged time to conventional cancer treatment. 


\section{Limitations}

213 The present study has several limitations. First, the rate and pattern of CAM use

214 at our center might be different from those in the general population, as we only

215 evaluated patients who attended hospital appointments, which would exclude patients

216 who select CAM treatments without seeking in-hospital treatment. It is also possible that

217 the use of face-to-face interviews could have influenced the participants' responses

218 (e.g., based on a desire to not reveal that they had used CAM treatments). Second,

219 various factors could influence the categorization of CAM treatments as herbal products

220 and diet supplements, especially as the ingredients were self-reported and their

221 accuracy is reliant on the patient's recall. Moreover, traditional medicine practitioners

222 tend to not disclose their formulations, which resulted in a large number of unlabeled

223 products containing unidentifiable ingredients. Third, there remains the possibility that

224 the study might not completely include other factors which could influence the time until

225 treatment (e.g., religions and cultural perspectives). Finally, caution is warranted in

226 inferring a causal relationship between CAM use and time to conventional treatment

227 because data were obtained from a cross-sectional study. 


\section{5. Conclusions}

Our findings demonstrated that there is a relatively moderate prevalence of CAM

231 use among Thai cancer patients, with most of the CAM treatments involving homemade

232 herbal products. The use of CAM was significantly associated with a prolonged time to 233 conventional treatment. Nevertheless, these findings do not imply that CAM should be

234 banned for all patients, although healthcare providers should recommend that patients

235 aim to use CAM treatments that are considered safe and will not interfere with

236 conventional treatments. 
239 Acknowledgments: We would like to thank Ms. Pimonwan promsuvan, Ms Pannee

240 Panjamnong, Mr Rangsan Chaikham, and operating room staff at Chonburi cancer

241 hospital for support in Data collection. Additionally, we are grateful to Dr. Akarathan

242 Jitnuyanont, the director of Chonburi cancer hospital, for his support and

243 encouragement. We would like to thank Editage (www.editage.com) for English

244 language editing.

245

246

247

248

249

250

251

252

253

254

255

256

257

258

259

260

261

262

263

264 
265

266

267

268

269

270

271

272

273

274

275

276

277

278

279

280

281

282

283

284

285

286

287

288

289

290

\section{References}

1.Rao C, Porapakkham Y, Pattaraarchachai J, Polprasert W, Swampunyalert N, Lopez A. Verifying causes of death in Thailand: rationale and methods for empirical investigation. Population Health Metrics. 2010;8:11.

2.Sriamporn S, Black R, Sankaranarayanan R, Kamsa-ad S, Parkin D, Vatanasapt $V$. Cancer survival in Khon Kaen province, Thailand. International Journal of Cancer. 1995;61(3):296-300.

3.Elit L. Wait times from diagnosis to treatment in cancer. Journal of Gynecologic Oncology. 2015;26(4):246-248.

4.Singh T, Pearlman PC, Kostelecky B. Supporting evidence-based national cancer control planning: the Asia-Pacific phase II leadership forum. Journal of Cancer Policy. 2017;12:75-78.

5.Paek SC, Meemon N, Wan TT. Thailand's universal coverage scheme and its impact on health-seeking behavior. Springerplus. 2016;5(1):1952.

6. Bhosai S, Sinthusake T, Miwa S, Bradley E. Factors affecting patient access in Thailand: understanding delay in care seeking for patients with cancer. Global Public Health. 2011;6(4):385-397.

7.World Health Organization. https://www.who.int/traditional-complementaryintegrative-medicine/about/en/. Published 2019. Accessed March 14, 2019

8.Bishop FL, Lewith GT. Who uses CAM? A narrative review of demographic characteristics and health factors associated with CAM use. Evidence-based Complementary and Alternative Medicine. 2008;7(1):11-28.

9.Adams J, Sibbritt D, Broom A, Loxton D, Pirotta M, Humphreys J, Lui C. A comparison of complementary and alternative medicine users and use across geographical areas: a national survey of 1,427 women. BMC Complementary and Alternative Medicine. 2011;11:85. 
291

292

293

294

295

296

297

298

299

300

301

302

303

304

305

306

307

308

309

310

311

312

313

10.Ellison CG, Bradshaw M, Roberts CA. Spiritual and religious identities predict the use of complementary and alternative medicine among US adults.

Preventive Medicine. 2011;54(1):9-12

11.Nahin RL, Dahlhamer JM, Stussman BJ. Health need and the use of alternative medicine among adults who do not use conventional medicine. BMC Health Services Research. 2010;10:220.

12.Poonthananiwatkul B, Lim R, Howard R, Pibanpaknitee P, Williamson E. Traditional medicine use by cancer patients in Thailand. Journal of Ethnopharmacology. 2015;168:100-107.

13.Ben-Arye E, Samuels N, Goldstein L, Mutafoglu K, Omran S, Schiff E, Charalambous H, Dweikat T, Ghrayeb IBar-Sela G, Turker I, Hassan A, Hassan E, Saad B, Nimri O, Kebudi R, Silbermann M. Potential risks associated with traditional herbal medicine use in cancer care: A study of Middle Eastern oncology health care professionals. Cancer. 2015;122(4):598-610.

14.Ohnishi S, Takeda H. Herbal medicines for the treatment of cancer chemotherapy-induced side effects. Frontiers in Pharmacology. 2015;6:14.

15. Ulbricht C, Chao W, Costa D, Rusie-Seamon E, Weissner W, Woods J. Clinical evidence of herb-drug interactions: a systematic review by the Natural Standard Research Collaboration. Current Drug Metabolism. 2008;9(10):1063-1120.

16.Kerdpon D. Factors related to advanced stage oral squamous cell carcinoma in southern Thailand. Oral Oncology. 2001;37(3):216-221.

17. Horneber M, Bueschel G, Dennert G, Less D, Ritter E, Zwahlen M. How many cancer patients use complementary and alternative medicine: a systematic review and metaanalysis. Integrative cancer therapies. 2011;11(3):187-203.

18. Tangcharoensathien V, Witthayapipopsakul W, Panichkriangkrai W, Patcharanarumol W, Mills A. Health systems development in Thailand: a solid platform for successful implementation of universal health coverage. The Lancet. 2018;391(10126):1205-1223. 
319

320

321

322

323

19.Gildea TR, DaCosta Byfield S, Hogarth DK, Wilson DS, Quinn CC. A retrospective analysis of delays in the diagnosis of lung cancer and associated costs. ClinicoEconomics and Outcomes Research. 2017;9:261-269.

20.Pocaphan P, Kondo S. Bacterial contamination assay of Thai herbal products. Journal of the Medical Association of Thailand. 2011;94(supplement 7):S162S165.

21.Mohd Mujar NM, Dahlui M, Emran NA, Abdul Hadi I, Wai YY, Arulanantham S, Hooi CC, Mohd Taib NA. Complementary and alternative medicine (CAM) use and delays in presentation and diagnosis of breast cancer patients in public hospitals in Malaysia. PLoS One. 2017;12(4):e0176394.

22.Malik I, Gopalan S. Use of CAM results in delay in seeking medical advice for breast cancer. European Journal of Epidemiology. 2002;18(8):817-822.

23.Johnson S, Park H, Gross C, Yu J. Complementary medicine, refusal of conventional cancer therapy, and survival among patients with curable cancers. JAMA Oncology. 2018;4(10):1375-1381.

24.Puataweepong $P$, Sutheechet N, Ratanamongkol P. A survey of complementary and alternative medicine use in cancer patients treated with radiotherapy in Thailand. Evidence-based Complementary and Alternative Medicine. 2012;2012:670408.

25.Gross A, Liu Q, Bauer-Wu S. Prevalence and predictors of complementary therapy use in advanced-stage breast cancer patients. Journal of Oncology Practice. 2007;3(6):292-295.

26. Bold J, Leis A. Unconventional therapy use among children with cancer in Saskatchewan. Journal of Pediatric Oncology Nursing. 2001;18(1):16-25.

27.Kelly K, Jacobson J, Kennedy D, Braudt S, Mallick M, Weiner M. Use of unconventional therapies by children with cancer at an urban medical center. Journal of Pediatric Hematology/Oncology. 2000;22(5):412-416. 
346

347
28.Kappauf H, Leykauf-Ammon D, Bruntsch U, Horneber M, Kaiser G, Büschel Gallmeier W. Use of and attitudes held towards unconventional medicine by patients in a department of internal medicine / oncology and haematology. Supportive Care in Cancer. 2000;8(4):314-322.

29.Münstedt K, Kirsch K, Milch W, Sachsse S, Vahrson H. Unconventional cancer therapy-survey of patients with gynaecological malignancy. Archives of Gynecology and Obstetrics. 1996;258(2):81-88.

30.Serafini M, Stanzione A, Foddai S, Anton R, Delmulle L. The European Role on Traditional Herbal Medicinal Products and Traditional Plant Food Supplements. Journal of Clinical Gastroenterology. 2012;46:S93-S94.

31.Pilkington K, Boshnakova A. Complementary medicine and safety: A systematic investigation of design and reporting of systematic reviews. Complementary Therapies in Medicine. 2012;20(1-2):73-82.

32.Izzo A, Ernst E. Interactions between herbal medicines and prescribed drugs. Drugs. 2009;69(13):1777-1798. 


\section{Table 1 (on next page)}

Demographic and clinical characteristics of patients with and without the use of complementary and alternative medicine 
Table 1: Demographic and clinical characteristics of patients with and without the use of complementary and alternative medicine

\begin{tabular}{|c|c|c|c|}
\hline Characteristics & Use & Non-use & P-value \\
\hline \multicolumn{4}{|l|}{ Sex } \\
\hline Male & $115(59.9 \%)$ & $107(45.7 \%)$ & 0.004 \\
\hline Female & $77(40.1 \%)$ & $127(54.3 \%)$ & \\
\hline \multicolumn{4}{|l|}{ Ages in years } \\
\hline$<45$ & $47(24.5 \%)$ & $44(18.8 \%)$ & 0.242 \\
\hline $45-65$ & $108(56.3 \%)$ & $133(56.8 \%)$ & \\
\hline$>65$ & $37(19.3 \%)$ & $57(24.4 \%)$ & \\
\hline
\end{tabular}

Cancer stage

\begin{tabular}{l|c|c|c} 
I-III & $106(55.2 \%)$ & $173(73.9 \%)$ & $<0.001$ \\
IV & $86(44.8 \%)$ & $61(26.1 \%)$ &
\end{tabular}

Household income

\begin{tabular}{c|c|c|c} 
Low $(<300$ US\$) & $87(45.3 \%)$ & $126(53.8 \%)$ & 0.080 \\
\hline High $(\geq 300$ US\$) & $105(54.7 \%)$ & $108(46.2 \%)$ & \\
\hline
\end{tabular}

Highest education

\begin{tabular}{|c|c|c|c|}
\hline Primary school & $137(71.4 \%)$ & $174(74.4 \%)$ & 0.487 \\
\hline Secondary school or higher & $55(28.6 \%)$ & $60(25.6 \%)$ & \\
\hline \multicolumn{4}{|l|}{ Cancer type } \\
\hline Breast cancer & $14(7.3 \%)$ & $41(17.5 \%)$ & 0.040 \\
\hline Gynecological cancer & $34(17.7 \%)$ & $37(15.8 \%)$ & \\
\hline Head and neck cancer & $82(42.7 \%)$ & $85(36.3 \%)$ & \\
\hline Colorectal cancer & $31(16.1 \%)$ & $36(15.4 \%)$ & \\
\hline Others & $31(16.1 \%)$ & $35(15.0 \%)$ & \\
\hline \multicolumn{4}{|l|}{ Referral province } \\
\hline Nearby a & $138(71.9 \%)$ & $172(73.5 \%)$ & 0.707 \\
\hline Distant ${ }^{b}$ & $54(28.1 \%)$ & $62(26.5 \%)$ & \\
\hline \multicolumn{4}{|l|}{ Treatment } \\
\hline Surgery & $61(31.8 \%)$ & $85(36.3 \%)$ & 0.604 \\
\hline Chemotherapy & $56(29.2 \%)$ & $62(26.5 \%)$ & \\
\hline Radiotherapy & $75(39.0 \%)$ & $87(37.2 \%)$ & \\
\hline
\end{tabular}

a Nearby areas were defined as the Chonburi, Rayong, and Chachoengsao provinces (Article S2).

b Distant areas included Chantaburi, Trat, Sa Kaeo, Prachinburi, Samut Prakan, and other provinces outside the Eastern region (Article S2).

1 


\section{Table 2 (on next page)}

Complementary and alternative medicines that were reported by the 192 patients 
Table 2: Complementary and alternative medicines that were reported by the 192 patients ${ }^{a}$

\begin{tabular}{|c|c|c|c|}
\hline Medicine & $\begin{array}{c}\text { Number of use } \\
(\%)\end{array}$ & Type & Major ingredients \\
\hline $\begin{array}{l}\text { Unlabeled fresh and } \\
\text { processed herbal products }\end{array}$ & $74(34.3 \%)$ & Homemade product & Unknown \\
\hline "Ya Mor Sang" & $41(20.0 \%)$ & Homemade product & $\begin{array}{l}\text { Rice hulls and various } \\
\text { Thai herbs }\end{array}$ \\
\hline \multirow[t]{5}{*}{ Lingzhi mushroom } & $35(16.2 \%)$ & All & Ganoderma lucidum \\
\hline & 6 & Homemade product & \\
\hline & 17 & Registered diet supplement & \\
\hline & 5 & Registered herbal medicine & \\
\hline & 8 & Undetermined product & \\
\hline Porcupine flower & $9(4.2 \%)$ & Homemade product & Barleria prionitis \\
\hline "G-herb" & $7(3.2 \%)$ & Registered herbal medicine & Various Thai herbs \\
\hline Soursop tea & $7(3.2 \%)$ & Homemade product & Annona muricata $L$ \\
\hline Dong-ChongXiaCao & $7(3.2 \%)$ & Registered diet supplement & Ophiocordyceps sinensis \\
\hline "Nan Chao Woei" & $5(2.3 \%)$ & Homemade product & $\begin{array}{l}\text { Gymnanthemum } \\
\text { extensum }\end{array}$ \\
\hline Crocodile blood capsule & $4(1.9 \%)$ & Registered diet supplement & $\begin{array}{l}\text { Freeze-dried crocodile } \\
\text { blood }\end{array}$ \\
\hline Sesame extracts & $4(1.9 \%)$ & Registered diet supplement & Sesamum indicum \\
\hline "Plu Kaow" & $3(1.4 \%)$ & Registered herbal medicine & Houttuynia cordata \\
\hline Mangosteen juice & $3(1.4 \%)$ & Registered diet supplement & Garcinia mangostana \\
\hline $\begin{array}{l}\text { Commercial fruit and } \\
\text { vegetable extract beverage }\end{array}$ & $2(0.9 \%)$ & Registered diet supplement & $\begin{array}{l}\text { Various fruits and } \\
\text { vegetables }\end{array}$ \\
\hline "Bai Ya Nang" & $2(0.9 \%)$ & Registered diet supplement & Tiliacora triandra \\
\hline Aloe vera juice & $1(0.5 \%)$ & Registered diet supplement & Aloe vera \\
\hline
\end{tabular}




\begin{tabular}{|c|c|c|c|}
\hline "Kao yen” & $1(0.5 \%)$ & Homemade product & Smilacaceae \\
\hline "Yong-Heng herbal solution" & $1(0.5 \%)$ & Registered herbal medicine & Various Chinese herbs \\
\hline “Luk Tai Bai” & $1(0.5 \%)$ & Homemade product & Phyllanthus niruri \\
\hline "Prai" & $1(0.5 \%)$ & Homemade product & Zingiber cassumunar \\
\hline "Sing Mo La" & $1(0.5 \%)$ & Registered herbal medicine & Cyrtosperma johnstonii \\
\hline Turmeric capsule & $1(0.5 \%)$ & Registered herbal medicine & Curcuma longa \\
\hline Abalone mushroom & $1(0.5 \%)$ & Registered diet supplement & Pleurotus ostreatus \\
\hline "Pien Tze Huang" & $1(0.5 \%)$ & Registered herbal medicine & $\begin{array}{l}\text { Traditional Chinese } \\
\text { formulation }\end{array}$ \\
\hline Korean ginseng & $1(0.5 \%)$ & Homemade product & Panax ginseng \\
\hline Soapberry tree & $1(0.5 \%)$ & Homemade product & Sapindus trifoliatus $L$. \\
\hline Fish oil & $1(0.5 \%)$ & Registered diet supplement & - \\
\hline Massage & $1(0.5 \%)$ & - & - \\
\hline
\end{tabular}

$1{ }^{a}$ A total of 24 patients reported using more than one type of complementary and alternative medicine. 


\section{Table 3 (on next page)}

The associations between times until conventional treatment and various factors 
Table 3: The associations between times until conventional treatment and various factors

\begin{tabular}{|c|c|c|c|c|c|c|c|c|c|c|c|c|c|}
\hline \multirow[t]{2}{*}{ Factors } & \multirow[t]{2}{*}{$\mathbf{N}$} & \multicolumn{2}{|c|}{$\begin{array}{l}\text { Wait time } \\
\text { (days) }\end{array}$} & \multicolumn{5}{|c|}{ Univariable analysis } & \multicolumn{5}{|c|}{ Multivariable analysis a } \\
\hline & & Median & IQR & Coef $b$ & $95 \% \mathrm{Cl}$ & Beta & $\mathbf{R}^{2}$ & $\mathrm{P}$-value & Coef ${ }^{b}$ & $95 \% \mathrm{Cl}$ & Beta & $\mathbf{R}^{2}$ & P-value \\
\hline
\end{tabular}

\begin{tabular}{|c|c|c|c|c|c|c|c|c|c|c|c|c|c|}
\hline \multicolumn{14}{|l|}{ Sex } \\
\hline Male & 222 & 51 & $35.0-90.0$ & 0 & & & & & & & & & \\
\hline Female & 204 & 49.5 & $31.0-88.0$ & -7.2 & $-36.7,22.2$ & -0.023 & 0.001 & 0.630 & - & - & - & - & - \\
\hline
\end{tabular}

\begin{tabular}{|c|c|c|c|c|c|c|c|c|c|c|c|c|c|}
\hline \multicolumn{14}{|l|}{ Cancer stage } \\
\hline$|-|||$ & 279 & 47.5 & $32.5-79.5$ & 0 & & & & & 0 & & & & \\
\hline IV & 147 & 59 & $35.0-114.0$ & 42.1 & $11.4-72.7$ & 0.130 & 0.015 & 0.007 & 38.9 & $8.4-69.5$ & 0.140 & 0.140 & 0.013 \\
\hline \multicolumn{14}{|c|}{ Complementary and alternative medicine } \\
\hline Non-use & 234 & 44 & $30.0-69.0$ & 0 & & & & & 0 & & & & \\
\hline Any use & 192 & 63 & $38.5-119.5$ & 70.3 & $41.5-99.0$ & 0.228 & 0.052 & $<0.001$ & 56.3 & $27.9-84.6$ & 0.215 & 0.140 & $<0.001$ \\
\hline Use before $T_{x}$ & 139 & 79 & $43.0-157.0$ & 98.1 & $65.3-131.0$ & 0.083 & 0.086 & $<0.001$ & 80.4 & $48.1-112.6$ & 0.284 & 0.180 & $<0.001$ \\
\hline Use after $T_{x}$ & 53 & 39 & $34.0-62.0$ & -0.8 & $-17.0,15.5$ & -0.005 & 0 & 0.926 & - & - & - & - & - \\
\hline
\end{tabular}

Conventional treatment modalities

\begin{tabular}{|c|c|c|c|c|c|c|c|c|c|c|c|c|c|}
\hline Surgery & 146 & 41 & $29.0-62.0$ & 0 & & & & & 0 & & & & \\
\hline Chemotherapy & 118 & 39 & $28.0-64.0$ & 24.1 & $-11.3,59.6$ & 0.083 & 0.007 & 0.181 & - & - & - & - & - \\
\hline Radiotherapy & 162 & 78 & $49.5-112.5$ & 59.6 & $30.8-88.4$ & 0.229 & 0.053 & $<0.001$ & 51.3 & $23.3-79.3$ & 0.197 & 0.140 & $<0.001$ \\
\hline
\end{tabular}

$$
\text { Income }
$$

\begin{tabular}{|c|c|c|c|c|c|c|c|c|c|c|c|c|c|}
\hline $\operatorname{High}(\geq 300$ US $\$$ ) & 213 & 47 & $30.0-80.5$ & 0 & & & & & 0 & & & & \\
\hline Low (<300 US $\$)$ & 213 & 54.5 & $35.5-95.0$ & 17.2 & $-12.1,46.6$ & 0.056 & 0.003 & 0.250 & 25.2 & $-6.7,57.2$ & 0.097 & 0.140 & 0.121 \\
\hline
\end{tabular}

Education

\begin{tabular}{|c|c|c|c|c|c|c|c|c|c|c|c|c|c|}
\hline Higher education & 115 & 49 & $30.0-85.0$ & 0 & & & & & 0 & & & & \\
\hline Primary school & 311 & 50.5 & $34.0-90.0$ & -2.5 & $-35.7,30.8$ & -0.007 & 0 & 0.884 & 5.5 & $-30.5,41.4$ & 0.018 & 0.140 & 0.765 \\
\hline \multicolumn{14}{|l|}{ Referral areas } \\
\hline Nearby area ${ }^{c}$ & 310 & 48 & $33.0-88.0$ & 0 & & & & & 0 & & & & \\
\hline
\end{tabular}




\begin{tabular}{|c|c|c|c|c|c|c|c|c|c|c|c|c|c|}
\hline Distant area ${ }^{d}$ & 116 & 53.5 & $36.0-90.0$ & -0.7 & $-33.8,32.5$ & -0.002 & 0 & 0.969 & -3.5 & $-34.5,27.5$ & -0.012 & 0.140 & 0.825 \\
\hline \multicolumn{14}{|l|}{ Health benefits } \\
\hline Other ${ }^{\mathrm{e}}$ & 124 & 46 & $30.0-89.0$ & 0 & & & & & 0 & & & & \\
\hline UCS & 302 & 52.5 & $34.5-89.0$ & -8.5 & $-40.9,23.9$ & -0.025 & 0.001 & 0.607 & -10.0 & $-43.6,23.7$ & -0.035 & 0.140 & 0.560 \\
\hline
\end{tabular}

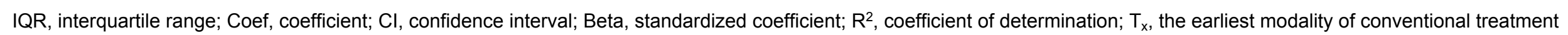
for each patient; UCS, universal coverage scheme

a The analyses were adjusted for all of the other factors included in the multivariable analysis.

$\mathrm{b}$ The coefficient represents the additional days of waiting for each category relative to the reference category.

c Nearby areas included the Chonburi, Rayong, and Chachoengsao provinces (Article S2).

d Distant areas included Chantaburi, Trat, Sa Kaeo, Prachinburi, Samut Prakan, and other provinces outside the Eastern region (Article S2).

e Other health benefit schemes included civil-servant, social security, and self-pay schemes.

\section{1}

